\title{
Erratum to: Specially Protected Natural Areas of the Kaliningrad Region
}

\author{
I.I. Volkova, T.V. Shaplygina, and E.S. Bubnova
}

V.A. Gritsenko et al. (eds.), Terrestrial and Inland Water Environment of the Kaliningrad Region - Environmental Studies in the Kaliningrad Region, Hdb Env Chem, DOI 10.1007/698_2017_98,

C) Springer International Publishing AG 2017

\section{Erratum to: Hdb Env Chem 10.1007/698_2017_98}

Inadvertently, the second affiliation of the co-author E.S. Bubnova was mentioned incorrectly in this chapter. This has now been updated.

The updated online version for this chapter can be found under DOI 10.1007/698_2017_98

I.I. Volkova ( $ه)$ and T.V. Shaplygina

Department of Geography, Nature Management and Spatial Development, Immanuel Kant Baltic Federal University, Kaliningrad, Russia

e-mail: volkova.bfu@yandex.ru; tshaplygina@gmail.com

E.S. Bubnova

Immanuel Kant Baltic Federal University, Kaliningrad, Russia

P.P. Shirshov Institute of Oceanology, Russian Academy of Sciences, 36, Nakhimovsky Pr., Moscow 117997, Russia

e-mail: bubnova.kat@gmail.com 